\title{
Elastic Orbital Angular Momentum
}

\author{
G. J. Chaplain $\oplus^{1,{ }^{1}}$ J. M. De Ponti® ${ }^{2}$ and R. V. Craster $\oplus^{3,4,5}$ \\ ${ }^{1}$ Electromagnetic and Acoustic Materials Group, Department of Physics and Astronomy, University of Exeter, \\ Exeter EX4 4QL, United Kingdom \\ ${ }^{2}$ Department of Civil and Environmental Engineering, Politecnico di Milano, Piazza Leonardo da Vinci, 32, 20133 Milano, Italy \\ ${ }^{3}$ Department of Mathematics, Imperial College London, 180 Queen's Gate, South Kensington, London SW7 2AZ, United Kingdom \\ ${ }^{4}$ Department of Mechanical Engineering, Imperial College London, London SW7 2AZ, United Kingdom \\ ${ }^{5}$ UMI 2004 Abraham de Moivre-CNRS, Imperial College London, London SW7 2AZ, United Kingdom
}

(Received 13 October 2021; accepted 13 January 2022; published 11 February 2022)

\begin{abstract}
We identify that flexural guided elastic waves in elastic pipes carry a well-defined orbital angular momentum associated with the compressional dilatational potential. This enables the transfer of elastic orbital angular momentum, that we numerically demonstrate, through the coupling of the compressional potential in a pipe to the acoustic pressure field in a surrounding fluid in contact with the pipe.
\end{abstract}

DOI: 10.1103/PhysRevLett.128.064301

Introduction.-Some thirty years ago, the seminal work of Allen et al. [1] demonstrated that Laguerre-Gaussian (LG) laser modes carry a well-defined orbital angular momentum (OAM), per quanta of light, about the beam axis. Crucially they outlined how such OAM, related to the spatial distribution of the laser field [2], can be extracted and converted into a mechanical torque [3] and that its existence arises physically due to the helical wave-front structure associated with a central phase singularity [4]. This differed from previous measurements of the torque exerted by the transfer of spin angular momentum associated with polarization $[5,6]$. These significant findings drove unabated interest in this previously neglected mechanical property of light [7-9], and have led to a renaissance in optical tweezers [10-16].

Perhaps the most distinct classical wave system from electromagnetism is elasticity; elastic materials are governed by constitutive relations that invoke a rank 4 stiffness tensor, and even in their simplest isotropic form they support two elastic waves (compression and polarized shear) that travel within the bulk at distinct wave speeds; these become inherently coupled upon reflection from a surface. Mirroring the timeline of research in optical OAM, only recently has the intrinsic spin of elastic waves been studied [17], with elastic OAM being largely neglected-it has only been considered in association with the phase of coupled waveguides [18], or presented canonically in conjunction with the energy-momentum tensor for elasticity, the Eshelby tensor [19-22].

In this Letter this disparity is addressed. We focus entirely on the OAM of elastic waves with inclined phase fronts, demonstrating that it is the scalar dilatational potential that carries a well-defined elastic OAM. The natural setting for such guided waves is along hollow elastic pipes. We consider flexural modes along pipes, leveraging the fact they can be excited using an elastic analog to the spiral phase plate (Fig. 1), and show that the transfer of elastic OAM is possible in fluid-solid coupled systems, providing motivation towards applications for acoustic tweezers, microfluidic devices, and nondestructive evaluation.

To unequivocally show that elastic OAM is carried by mechanical waves in pipes, we first outline the form of the canonical angular momentum density by its relation to mechanical energy flux. We derive here, from first principles, this relation from the Eshelby tensor [20].

OAM in elasticity.-Waves in an isotropic, homogeneous linear elastic material obey the dynamic Navier-Cauchy equation [23]

$$
\mu \partial_{j} \partial_{j} \xi_{i}+(\lambda+\mu) \partial_{j} \partial_{i} \xi_{i}=\rho \ddot{\xi}_{i}
$$

with $\xi_{i}$ the displacement and $\ddot{\xi}_{i}$ its double time derivative. Lamé's first and second parameters are denoted $\lambda, \mu$,

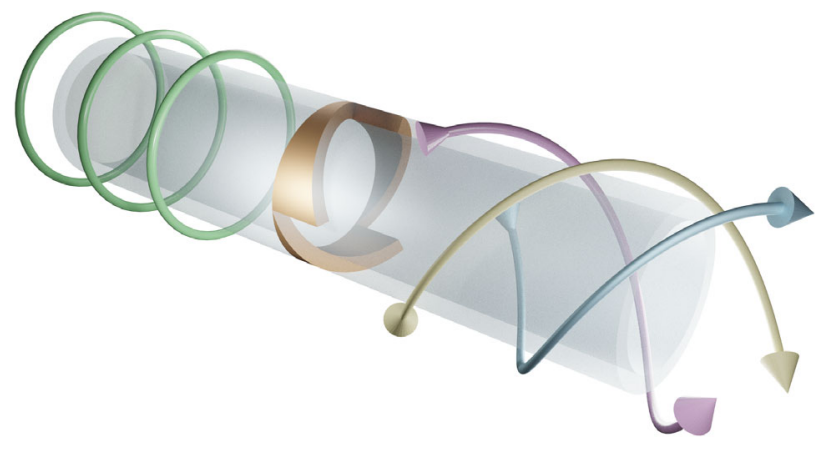

FIG. 1. Schematic of an elastic spiral phase pipe (copper region) in a hollow elastic pipe (transparent region). Purely longitudinal waves, e.g., $L(0,2)$ modes (circular phase fronts), are mode converted into flexural $F(3, n)$ waves (helical phase fronts). 
respectively. In this coordinate-free index notation we adopt the Einstein summation convention throughout. The linear constitutive law governing such a material is

$$
\sigma_{i j}=C_{i j k l} \varepsilon_{k l}=\lambda \delta_{i j} \varepsilon_{k k}+2 \mu \varepsilon_{i j},
$$

where $\sigma_{i j}$ is the stress tensor, $C_{i j k l}$ is the stiffness tensor, and $\varepsilon_{i j} \equiv \frac{1}{2}\left(\xi_{i, j}+\xi_{j, i}\right)$ is the strain tensor (comma notation denotes partial differentiation). The elastodynamic equations (1) are, of course, recovered by the Euler-Lagrange equations that dictate the vanishing of the variational derivative

$$
\frac{\delta \mathcal{L}}{\delta \xi_{j}} \equiv \frac{\partial \mathcal{L}}{\partial \xi_{j}}-\frac{\partial}{\partial t}\left(\frac{\partial \mathcal{L}}{\partial \dot{\xi}_{j}}\right)=0
$$

with $\mathcal{L}$ being the Lagrangian density for elastic waves given by

$$
\mathcal{L}=\frac{1}{2} \rho \dot{\xi}_{i} \dot{\xi}_{i}-\frac{1}{2} C_{i j k l} \xi_{i ; j} \xi_{k ; l},
$$

where semicolon notation denotes covariant differentiation and $\mathcal{L}=\mathcal{L}\left(\xi_{i}, \xi_{i, j}, \boldsymbol{x}, t\right)$ with $\boldsymbol{x}$ the position vector. The Eshelby tensor results from the canonical procedure for constructing stress-energy tensors, following Noether's theorem, and is given as [24]

$$
T_{l j}=\mathcal{L} \delta_{l j}-\frac{\partial \mathcal{L}}{\partial \xi_{i, j}} \xi_{i, l} .
$$

From this the energy density, $U=T_{00}$, and flux, $F_{j}=T_{0 j}$, of the elastic waves can be constructed:

$$
\begin{aligned}
U & =\frac{\partial \mathcal{L}}{\partial \dot{\xi}_{i}} \dot{\xi}_{i}-\mathcal{L}=\frac{1}{2} \rho \dot{\xi}_{i} \dot{\xi}_{i}+\frac{1}{2} C_{i j k l} \xi_{i ; j} \xi_{k ; l,}, \\
F_{j} & =\frac{\partial \mathcal{L}}{\partial \xi_{i ; j}} \dot{\xi}_{i}=-C_{i j k l} \dot{\xi}_{i} \xi_{k ; l} .
\end{aligned}
$$

Thus we have arrived at the mechanical analog of the Poynting vector through the mechanical energy flux, $F_{j}$. Herein we assume time harmonicity such that $\dot{\xi}_{k}=-i \omega \xi_{k}$ with $\omega$ being the radian frequency. Therefore, the timeaveraged complex mechanical energy flux density, which can be considered the Poynting vector density of elastic waves [17], is written as

$$
F_{j}=-\frac{1}{2} \mathfrak{R e}\left(\sigma_{j i} \dot{\xi}_{i}^{*}\right)=-\frac{\omega}{2} \mathfrak{I m}\left(\sigma_{j i} \xi_{i}^{*}\right),
$$

where $*$ denotes complex conjugation. The integral of this quantity, as in electromagnetism, is thus interpreted as the linear momentum density.
The flux of the corresponding angular momentum density is defined by the rank 3 tensor $M_{i j k}=x_{i} T_{j k}-$ $x_{j} T_{i k}$ [7]. The antisymmetric pseudotensor of rank 2, $M_{i j 0}=\epsilon_{i l m} x_{l} T_{m j}$ has spatial components, i.e., the pseudovector $M_{i}=1 / 2 \epsilon_{i j k} M_{j k 0}$ [2] that are then the familiar angular momentum density of the form, in vector notation, $\boldsymbol{M}=\boldsymbol{r} \times\langle\boldsymbol{p}\rangle$ with $\langle\boldsymbol{p}\rangle$ the time-averaged linear momentum density. For convenience we now switch from index notation to coordinate dependent vector notation and explicitly consider cylindrical polar coordinates.

Analogous to the treatment of electromagnetic waves in Ref. [1] we now consider the elastic angular momentum density as

$$
\boldsymbol{M}=-\frac{\omega}{2} \mathfrak{J} \mathfrak{m}\left[\boldsymbol{r} \times\left(\underline{\underline{\sigma}} \cdot \xi^{*}\right)\right],
$$

highlighting the tensorial nature of the stress tensor with a double underline, $\underline{\sigma}$, such that the total angular momentum is then

$$
\mathcal{J}=-\frac{\omega}{2} \mathfrak{I m} \int \boldsymbol{r} \times\left(\underline{\underline{\sigma}} \cdot \xi^{*}\right) d \boldsymbol{r}
$$

The complex displacement field $\xi$ is separated into longitudinal and transverse components via Helmholtz decomposition. These are written, respectively, in terms of the curl-less dilatational scalar potential, $\Phi$ (analogous to the scalar potential of the LG beams in optics, see Supplemental Material [25]), and the divergenceless equivoluminal vector shear potential, $\Psi$, such that

$$
\boldsymbol{\xi}=\boldsymbol{\xi}_{L}+\boldsymbol{\xi}_{T}=\nabla \Phi+\nabla \times \boldsymbol{\Psi}
$$

where $\boldsymbol{\xi}_{L}$ and $\boldsymbol{\xi}_{T}$ denote the longitudinal and transverse parts, respectively. Using this, the elastodynamic equations (1) reduce to two wave equations for compressional and shear waves:

$$
\begin{aligned}
\nabla^{2} \Phi & =c_{p}^{-2} \ddot{\Phi}, & c_{p} & =\sqrt{\frac{\lambda+2 \mu}{\rho}}, \\
\nabla^{2} \Psi & =c_{s}^{-2} \ddot{\Psi}, & c_{s} & =\sqrt{\frac{\mu}{\rho}},
\end{aligned}
$$

with $c_{p}$ and $c_{s}$ being the compressional and shear bulk wave speeds, respectively.

The angular momentum density can therefore be rewritten in terms of its spin, orbit, and "additional" components [22], each with individual contributions from the shear and compressional potentials. Long et al. [17] identify this additional component as hybrid orbital and spin Poynting densities.

The energy flux density (7) can then be split into orbital components which take the form 


$$
\begin{aligned}
\boldsymbol{p}_{L}^{o} & =\frac{\omega \rho}{2} c_{p}^{2} \mathfrak{\Im} \mathfrak{m}\left[\left(\boldsymbol{\xi}_{L}^{*} \cdot \nabla\right) \boldsymbol{\xi}_{L}\right], \\
\boldsymbol{p}_{T}^{o} & =\frac{\omega \rho}{2} c_{s}^{2} \mathfrak{\Im m}\left[\left(\boldsymbol{\xi}_{T}^{*} \cdot \nabla\right) \boldsymbol{\xi}_{T}\right], \\
\boldsymbol{p}_{H}^{o} & =\frac{\omega \rho}{2} c_{p}^{2} \mathfrak{\Im} \mathfrak{m}\left[\left(\boldsymbol{\xi}_{T}^{*} \cdot \nabla\right) \boldsymbol{\xi}_{L}\right]+\frac{\omega \rho}{2} c_{s}^{2} \mathfrak{\Im m}\left[\left(\boldsymbol{\xi}_{L}^{*} \cdot \nabla\right) \boldsymbol{\xi}_{T}\right],
\end{aligned}
$$

with the subscripts $L, T, H$ corresponding to the longitudinal, transverse (shear), and hybrid parts, respectively. We now prove that, for displacement fields with inclined phase fronts, the longitudinal part of the wave field $\boldsymbol{\xi}_{L}$, associated with compressional motion, carries a welldefined OAM.

Flexural waves in pipes serve as exemplar mode shapes capable of carrying elastic OAM. We consider elastic waves propagating along an infinitely long, hollow elastic cylinder with axis oriented in the $z$ direction of inner radius $r_{a}$ and outer radius $r_{b}$. The first general solution for these guided harmonic waves was derived by Gazis [26,27], who showed there are three families of modes: longitudinal, torsional, and flexural. The naming convention for such modes classifies these as $L(m, n), T(m, n)$, and $F(m, n)$, respectively [28]. Here $m$ denotes the circumferential order, or azimuthal index, with $n$ the group order. The mode shapes for which $m=0$ are axisymmetric, i.e., their angular profile is constant. We consider nonaxisymmetric flexural modes $F(m>0, n)$ whose mode shapes vary sinusoidally in the circumferential direction. Following the ansatz of Gazis, we leverage the cylindrical symmetry of the pipe and writing the coordinate system as $(\hat{\boldsymbol{r}}, \hat{\boldsymbol{\theta}}, \hat{z})$ pose the form of the scalar dilatational potential as

$$
\Phi=\phi(r) \exp \left[i\left(m \theta+k_{z} z-\omega t\right)\right] .
$$

The compressional displacement field then has the form

$$
\boldsymbol{\xi}_{L}=\left(\phi^{\prime}, \frac{i m \phi}{r}, i k \phi\right) \exp \left[i\left(m \theta+k_{z} z-\omega t\right)\right],
$$

with the prime notation denoting partial differentiation with respect to $r$. After substitution into (11) the radial profile $\phi(r)$ is solved by a linear combination of Bessel's functions, each with a complex amplitude. These coefficients are solved for by employing the infinitely long cylinder gauge, $\nabla \cdot \Psi=0$, and traction free boundary conditions on the inner and outer radii $\sigma_{r r}=\sigma_{r \theta}=\sigma_{r z}=\left.0\right|_{r_{a}, r_{b}}$ (see Supplemental Material [25]). As such the guided modes in elastic pipes can be thought of as Bessel "beams" in the sense that the radial distributions satisfy Bessel's equation.

The contribution of $\boldsymbol{p}_{L}^{o}$ to the OAM density along the pipe axis $\boldsymbol{M} \cdot \hat{z}$ is defined as $\boldsymbol{M}_{L}^{o} \cdot \hat{z}=r \boldsymbol{p}_{L}^{o} \cdot \hat{\boldsymbol{\theta}}$, where $\boldsymbol{p}_{L}^{o} \cdot \hat{\boldsymbol{\theta}}$ is the azimuthal component of the orbital, longitudinal part of the linear momentum density. To evaluate this quantity we are required to evaluate the advective terms that arise in (12) due to the variation of the Lagrangian basis vectors as the body deforms, highlighting its extrinsic nature; for an elastic deformation $\boldsymbol{\xi}, \boldsymbol{\alpha}$ is the Lagrangian position vector $\boldsymbol{\alpha}=\boldsymbol{\beta}-\boldsymbol{\xi}$ with $\boldsymbol{\beta}$ the Eulerian position vector after the deformation.

Calculation of the elastic OAM density along the pipe axis yields

$$
\begin{aligned}
\boldsymbol{M}_{L}^{o} \cdot \hat{z}= & m\left\{\frac { \omega \rho c _ { p } ^ { 2 } } { 2 } \left[\left|\phi^{\prime}\right|^{2}+\left(\frac{1}{r}\left(r \phi^{\prime \prime}+\phi^{\prime}\right)\right) \phi^{*}\right.\right. \\
& \left.\left.+\frac{\omega^{2}}{c_{p}^{2}}|\phi|^{2}-\frac{2}{r} \mathfrak{R e}\left(\phi \phi^{* \prime}\right)\right]\right\} .
\end{aligned}
$$

In general, $\phi(r)$ may be arbitrary and as such this result holds for all compressional wave fields with an azimuthally dependent profile, i.e., with inclined phase fronts, as is the case in electromagnetism [4]. Therefore, our assertion that the compressional component of the displacement field carries a well-defined elastic OAM is justified. The remaining contributions to the elastic OAM (from the transverse and hybrid components) also contain terms proportional to the azimuthal index $m$, but with additional factors (see Supplemental Material [25]) that leave them not fully quantized in the sense that they are only proportional to the azimuthal index. The physical significance of this for the exemplar case of guided waves in pipes is then that (i) trivially, $\boldsymbol{M}_{L}^{o} \cdot \hat{z}=0$ for both $L(0, n), T(0, n)$ modes which is to be expected for axisymmetric modes; (ii) pure flexural modes with a constant angular profile are required to carry OAM. Conventional means of exciting these modes in pipes rely on either complex arrangements of transducers (e.g., non-axisymmetric partial loading) or phased arrays [29-33]. Often many degenerate groups of flexural modes are excited simultaneously, including modes with both $\exp ( \pm i m \phi)$ components; the angular profile then changes with propagation distance due to modal superposition. As such there is zero average elastic OAM. Fortuitously, the recent advent of the elastic spiral phase pipe (eSPP), analogous to optical spiral phase plates [34,35], enables arbitrary $F(m, n)$ modes to be efficiently excited, via mode conversion, which boast a constant angular profile along the pipe axis.

We demonstrate in Fig. 2, via numerical calculation, that the elastic OAM associated with the dilatational potential for guided waves along a pipe carries a well-defined OAM. The associated orbital angular momentum flux density, at a constant plane in $z$, is given as $\mathcal{J}_{L} \hat{z}=-(\omega / 2) \mathfrak{I} \mathfrak{m}$ $\int \boldsymbol{M}_{L}^{o} \cdot \hat{z} d r d \theta$. For brevity we define $\mathcal{J}_{L z}=\mathcal{J}_{L} \cdot \hat{z}$. Figure 2(a) shows the dispersion curves for guided waves in an aluminium pipe, evaluated using a spectral collocation method [36-38] (corroborated with finite element computations [39], using the commercial software COMSOL Multiphysics [40] (B), described in the Supplemental Material [25]; the eigensolutions give frequency as the eigenvalue, with the corresponding eigenvector containing 
(a)



(b)

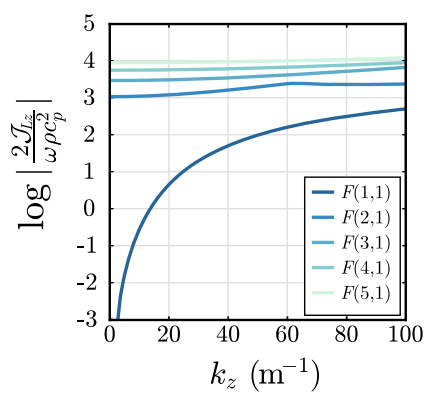

\begin{tabular}{llllll}
\multicolumn{9}{c}{$m$} \\
\hline 0 & 1 & 2 & 3 & 4 & 5
\end{tabular}

FIG. 2. (a) Dispersion curves of guided waves in an aluminium pipe of inner diameter $40 \mathrm{~mm}$ and thickness $10 \mathrm{~mm}$. Solid lines obtained by spectral collocation with points resulting from finite element computations. (b) Numerical evaluation (details reported in the Supplemental Material [25]) of the well-defined elastic OAM along the pipe axis, $\mathcal{J}_{L z}$, associated with the dilatational potential for the lowest curves of the flexural modes $F(m, 1)$.

the potential components $\left(\Phi, \Psi_{r}, \Psi_{\theta}, \Psi_{z}\right)$. These are used to numerically evaluate the ratio of the compressional OAM flux density to the energy flux density of a compressional bulk wave, $\log \left|\left(2 \mathcal{J}_{L z} / \omega \rho c_{p}^{2}\right)\right|$, shown in Fig. 2(b) for the lowest branches of the first five flexural modes $F(m=1 \ldots .5,1)$.

We now utilize an elastic spiral phase pipe (Fig. 1) to show that elastic OAM can be transferred to a fluid in contact with the elastic material; shear waves are not supported in fluids and as such only the compressional motion of the elastic material couples strongly to the acoustic pressure field in the fluid. The OAM transfer is observed by the introduction of rotational motion within the fluid, exciting spiraling acoustic wave fields.

OAM transfer.-The ability to transfer elastic OAM to a fluid is demonstrated numerically, via finite element simulations of an aluminium pipe partially submerged in water (Fig. 3). We excite a flexural $F(3,2)$ mode via mode conversion of a longitudinal $L(0,2)$ wave by passage through a suitably designed elastic spiral phase pipe (see Supplemental Material [25]). For a single frequency and wave vector, the compressional motion of the flexural mode within the pipe can be represented by a superposition of plane waves uniformly distributed over the circular aperture of the pipe. The plane wave components have mutual phases proportional to the azimuthal index $m$, endowed by the introduction of the eSPP. This compressional motion couples to the pressure field within the fluid at the submerged end of the pipe, producing rotating acoustic pressure fields. This is demonstrated in Figs. 3(b)-3(c) that show a snapshot in time of the dilatational field, through the trace of the strain tensor $\operatorname{tr}(\varepsilon)$, at the submerged pipe end and the pressure field within the fluid. The dilatation is related to the compressional potential through $\Phi=-(\nabla \cdot \boldsymbol{u}) / k_{z}^{2}=-\operatorname{tr}(\varepsilon) / k_{z}^{2}$. We only consider a partially submerged pipe in order to neglect Franz-type waves [41], and note that the OAM transfer is viewed via the mechanical torque the compressional motion enacts on the fluid, not by the generation of acoustic Bessel beams known to carry OAM [42-46].

Conclusions.-We have shown that elastic waves with inclined phase fronts can carry an extrinsic orbital angular momentum; it has been proved that the compressional dilatational potential carries a well-defined contribution, proportional to the azimuthal index $m$. This result is reminiscent of the case of LG beams in optics where the electromagnetic wave equation, under the paraxial approximation, is satisfied by a complex scalar function describing (a)

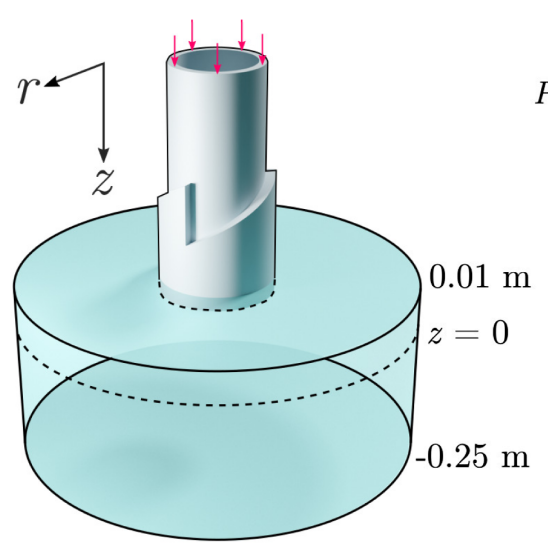

(b)

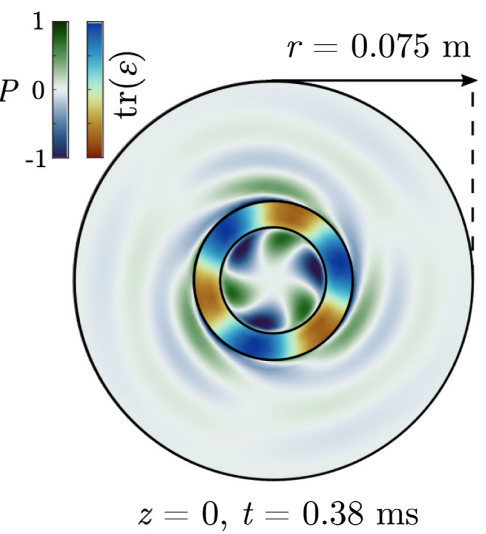

(c)

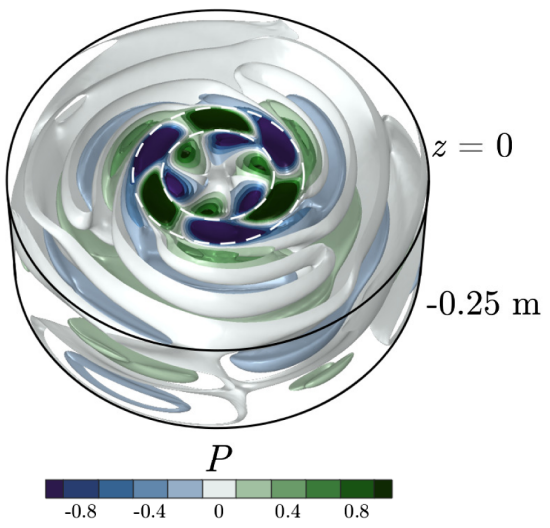

FIG. 3. Finite element time domain simulation of OAM transfer: (a) Schematic of the simulation domain, with pipe partially submerged $1 \mathrm{~cm}$ in water, surrounded by air. Arrows show axisymmetric longitudinal excitation position, with absorbing boundaries on exterior fluid walls. (b) Normalized compressional field in pipe [trace of the strain tensor, $\operatorname{tr}(\varepsilon)$ ] and pressure field $(P)$ in the fluid at the end of the pipe $(z=0)$. (c) Isosurfaces of fluid pressure in the region below the pipe showing spiraling acoustic waves, at the same time instance as in (b). Full details are shown in the Supplemental Material, along with frequency domain corroborations [25]. 
the field distribution, proportional to the azimuthal mode index. It is this phase profile that gives rise, in both cases, to the well-defined OAM.

The coupling of guided flexural waves in elastic pipes to acoustic pressure waves in fluids has been shown numerically through the compressional motion of the pipe. The implications are that the elastic OAM carried by the flexural modes can be transferred to acoustic pressure fields within a fluid. Inspired by the fact that optical LG laser modes have well-defined OAM, and that these modes are capable of being produced by spiral phase plates [47], we leverage recent developments in elastic spiral phase pipes to generate the desired flexural pipe modes. These eSPP enables efficient mode conversion of longitudinal modes to arbitrary flexural modes, crucially of a single handedness, i.e., that possess only one sign of $\exp ( \pm \operatorname{im} \theta)$. In this way the compressional motion in the pipe acts as a continuous phased acoustic pressure source in the fluid, opposed to conventional discrete acoustic sources [48]. Harnessing the mechanical torques associated with the elastic OAM then promises to unlock applications across acoustic tweezers, nondestructive testing, ultrasonic motor design, and acoustofluidic devices.

G. J. C. gratefully acknowledges financial support from the Royal Commission for the Exhibition of 1851 in the form of a Research Fellowship. J. M. De P. and R. V. C. acknowledge financial support from the H2020 FETproactive project MetaVEH under Grant Agreement No. 952039.

*g.j.chaplain@exeter.ac.uk

[1] L. Allen, M. W. Beijersbergen, R. J. C. Spreeuw, and J. P. Woerdman, Orbital angular momentum of light and the transformation of Laguerre-Gaussian laser modes, Phys. Rev. A 45, 8185 (1992).

[2] J. D. Jackson, Classical Electrodynamics (Wiley, New York, 1962).

[3] H. He, M. E. J. Friese, N.R. Heckenberg, and H. Rubinsztein-Dunlop, Direct Observation of Transfer of Angular Momentum to Absorptive Particles from a Laser Beam with a Phase Singularity, Phys. Rev. Lett. 75, 826 (1995).

[4] A. T. O'Neil, I. MacVicar, L. Allen, and M. J. Padgett, Intrinsic and Extrinsic Nature of the Orbital Angular Momentum of a Light Beam, Phys. Rev. Lett. 88, 053601 (2002).

[5] R. A. Beth, Mechanical detection and measurement of the angular momentum of light, Phys. Rev. 50, 115 (1936).

[6] J. H. Poynting, The wave motion of a revolving shaft, and a suggestion as to the angular momentum in a beam of circularly polarised light, Proc. R. Soc. A 82, 560 (1909).

[7] S. M. Barnett, Optical angular-momentum flux, J. Opt. B 4, S7 (2002).

[8] S. M. Barnett, Rotation of electromagnetic fields and the nature of optical angular momentum, J. Mod. Opt. 57, 1339 (2010).
[9] L. Allen, S. M. Barnett, and M. J. Padgett, Optical Angular Momentum (CRC Press, Boca Raton, 2016).

[10] V. Garcés-Chávez, D. McGloin, M. J. Padgett, W. Dultz, H. Schmitzer, and K. Dholakia, Observation of the Transfer of the Local Angular Momentum Density of a Multiringed Light Beam to an Optically Trapped Particle, Phys. Rev. Lett. 91, 093602 (2003).

[11] A. M. Yao and M. J. Padgett, Orbital angular momentum: Origins, behavior and applications, Adv. Opt. Photonics 3, 161 (2011).

[12] A. E. Willner, H. Huang, Y. Yan, Y. Ren, N. Ahmed, G. Xie, C. Bao, L. Li, Y. Cao, Z. Zhao et al., Optical communications using orbital angular momentum beams, Adv. Opt. Photonics 7, 66 (2015).

[13] K. Y. Bliokh and F. Nori, Transverse and longitudinal angular momenta of light, Phys. Rep. 592, 1 (2015).

[14] S. M. Barnett, M. Babiker, and M. J. Padgett, Optical orbital angular momentum, Phil. Trans. R. Soc. A 375, 20150444 (2017).

[15] M. J. Padgett, Orbital angular momentum 25 years on, Opt. Express 25, 11265 (2017).

[16] R. Chen, H. Zhou, M. Moretti, X. Wang, and J. Li, Orbital angular momentum waves: Generation, detection, and emerging applications, IEEE Commun. Surv. Tutorials 22, 840 (2019).

[17] Y. Long, J. Ren, and H. Chen, Intrinsic spin of elastic waves, Proc. Natl. Acad. Sci. U.S.A. 115, 9951 (2018).

[18] P. Deymier, K. Runge, J. Vasseur, A. Hladky, and P. Lucas, Elastic waves with correlated directional and orbital angular momentum degrees of freedom, J. Phys. B 51, 135301 (2018).

[19] J. D. Eshelby, The force on an elastic singularity, Phil. Trans. R. Soc. A 244, 87 (1951).

[20] J. Eshelby, The elastic energy-momentum tensor, J. Elast. 5, 321 (1975).

[21] K. O. Thielheim, Note on classical fields of higher order, Proc. Phys. Soc. London 91, 798 (1967).

[22] M. Lazar and H. O. Kirchner, The Eshelby stress tensor, angular momentum tensor and dilatation flux in gradient elasticity, Int. J. Solids Struct. 44, 2477 (2007).

[23] L. D. Landau and E. M. Lifshitz, Course of Theoretical Physics Vol. 7: Theory and Elasticity (Pergamon Press, New York, 1959).

[24] E. Noether, Invariant variation problems, Transp. Theory Stat. Phys. 1, 186 (1971).

[25] See Supplemental Material at http://link.aps.org/ supplemental/10.1103/PhysRevLett.128.064301 for more details on the theory of Laguerre-Gaussian beams, analytical and numerical solutions for guided waves in pipes.

[26] D. C. Gazis, Three-dimensional investigation of the propagation of waves in hollow circular cylinders. i. analytical foundation, J. Acoust. Soc. Am. 31, 568 (1959).

[27] D. C. Gazis, Three-dimensional investigation of the propagation of waves in hollow circular cylinders. II. Numerical results, J. Acoust. Soc. Am. 31, 573 (1959).

[28] M. Silk and K. Bainton, The propagation in metal tubing of ultrasonic wave modes equivalent to Lamb waves, Ultrasonics 17, 11 (1979).

[29] H. J. Shin and J. L. Rose, Guided waves by axisymmetric and non-axisymmetric surface loading on hollow cylinders, Ultrasonics 37, 355 (1999). 
[30] J. Li and J. L. Rose, Excitation and propagation of nonaxisymmetric guided waves in a hollow cylinder, J. Acoust. Soc. Am. 109, 457 (2001).

[31] J. Li and J. L. Rose, Angular-profile tuning of guided waves in hollow cylinders using a circumferential phased array, IEEE Trans. Ultrason. Ferroelectr. Freq. Control 49, 1720 (2002).

[32] J. L. Rose, Ultrasonic Guided Waves in Solid Media (Cambridge University Press, Cambridge, London, 2014).

[33] L. Tang and B. Wu, Excitation mechanism of flexuralguided wave modes $\mathrm{F}(1,2)$ and $\mathrm{F}(1,3)$ in pipes, J. Nondestruct. Eval. 36, 59 (2017).

[34] G. J. Chaplain and J. M. De Ponti, The elastic spiral phase pipe, J. Sound Vib. 523, 116718 (2022).

[35] M. Beijersbergen, R. Coerwinkel, M. Kristensen, and J. Woerdman, Helical-wavefront laser beams produced with a spiral phaseplate, Opt. Commun. 112, 321 (1994).

[36] A. Adamou and R. Craster, Spectral methods for modelling guided waves in elastic media, J. Acoust. Soc. Am. 116, 1524 (2004).

[37] F. H. Quintanilla, M. J. S. Lowe, and R. V. Craster, Modeling guided elastic waves in generally anisotropic media using a spectral collocation method, J. Acoust. Soc. Am. 137, 1180 (2015).

[38] B. Pavlakovic, M. Lowe, D. Alleyne, and P. Cawley, Disperse: A general purpose program for creating dispersion curves, in Review of Progress in Quantitative Nondestructive Evaluation (Springer, New York, 1997), pp. 185-192.

[39] A. Marzani, E. Viola, I. Bartoli, F. Lanza di Scalea, and P. Rizzo, A semi-analytical finite element formulation for modeling stress wave propagation in axisymmetric damped waveguides, J. Sound Vib. 318, 488 (2008).

[40] COMSOL Multiphysics ${ }^{\circledR}$ reference manual, version 5.6, www.comsol.com, COMSOL AB, Stockholm, Sweden, 2021.

[41] G. Frisk, J. Dickey, and H. Überall, Surface wave modes on elastic cylinders, J. Acoust. Soc. Am. 58, 996 (1975).

[42] B. T. Hefner and P. L. Marston, Acoustical helicoidal waves and laguerre-gaussian beams: Applications to scattering and to angular momentum transport, J. Acoust. Soc. Am. 103, 2971 (1998).

[43] R. Wunenburger, J. I. V. Lozano, and E. Brasselet, Acoustic orbital angular momentum transfer to matter by chiral scattering, New J. Phys. 17, 103022 (2015).

[44] Z. Y. Hong, J. Zhang, and B. W. Drinkwater, Observation of Orbital Angular Momentum Transfer from BesselShaped Acoustic Vortices to Diphasic Liquid-Microparticle Mixtures, Phys. Rev. Lett. 114, 214301 (2015).

[45] K. Y. Bliokh and F. Nori, Spin and orbital angular momenta of acoustic beams, Phys. Rev. B 99, 174310 (2019).

[46] I. D. Toftul, K. Y. Bliokh, M. I. Petrov, and F. Nori, Acoustic Radiation Force and Torque on Small Particles as Measures of the Canonical Momentum and Spin Densities, Phys. Rev. Lett. 123, 183901 (2019).

[47] S. S. R. Oemrawsingh, J. A. W. van Houwelingen, E. R. Eliel, J. P. Woerdman, E. J. K. Verstegen, J. G. Kloosterboer, and G. W.'t Hooft, Production and characterization of spiral phase plates for optical wavelengths, Appl. Opt. 43, 688 (2004).

[48] M. Cromb, G. M. Gibson, E. Toninelli, M. J. Padgett, E. M. Wright, and D. Faccio, Amplification of waves from a rotating body, Nat. Phys. 16, 1069 (2020). 